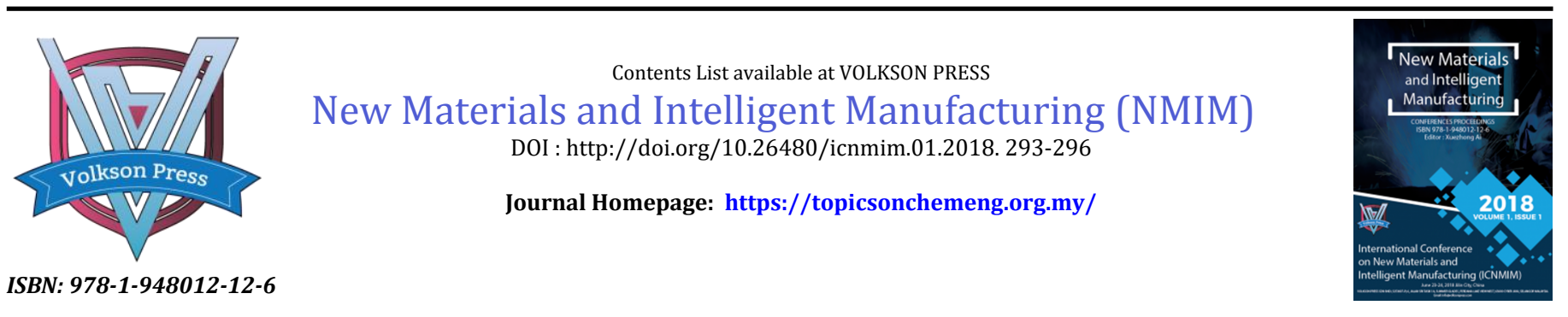

\title{
THE INFLUENCE OF MAGNETOSTRICTIVE TRANSDUCER RADIATION PLATE MATERIAL ON RADIATION SOUND FIELD DISTRIBUTION
}

\author{
Yu Wenxin ${ }^{1}$, Ding Yanchun ${ }^{2}$, Liu Haibo ${ }^{1}$ \\ ${ }^{1}$ Jilin Institute of Chemicak Technology, College of Mechanical and Electrical Engineering, Jilin 132022, China \\ ${ }^{2}$ College of Electro-mechanical Engineering, Changchun University of Science and Technology, Changchun 130022, China
}

This is an open access article distributed under the Creative Commons Attribution License, which permits unrestricted use, distribution, and reproduction in any medium, provided the original work is properly cited.

\section{ARTICLE DETAILS}

\section{Article History:}

Received 26 June 2018 Accepted 2 July 2018

Available online 1 August 2018

\begin{abstract}
The radiant panel is transducer of the sound source output device, the installation space constraints under the condition of enrollment size, increase the radiation plate vibration amplitude of the focus of research. This paper only considering the influence of the materials of radiant panel vibration amplitude and has carried on the finite element software WORKBENCH and ATILA software analyzes the magneto strictive transducer radiant panel material influence on vibration amplitude frequency characteristics and the influence of sound field distribution inside the cavity. Results show that the same incentives and boundary condition, the aluminum material appeared radiant panel piston type when the frequency is highest, has the biggest output displacement which was of 4.48 to 24.8 times to the other materials panel; After joining the cavity body, aluminum radiant panel inside the cavity acoustic pressure amplitude is the largest and the panel radiated sound pressure amplitude is of 1.2 to 2.4 times than the other material. Above conclusion for frequency miniature transducer radiant panel provides a strong basis for the design of the work.
\end{abstract}

\section{KEYWORDS}

Transducer, Radiant panel materials, The vibration displacement, Frequency, Sound pressure amplitude, The finite element analysis.

\begin{abstract}
1.INTRODUCTION
The transducer is a device that performs energy, conversion between two systems. In recent years, with the rapid development of new technologies and new materials, provides powerful backing for transducer research, ultrasonic magnetostrictive transducers have been widely used in the ultrasonic field due to their high magnetostrictive coefficient, high compressive strength, high mechanical response speed, and high temperature resistance [1]. The radiation board is a sound source output device of a transducer, according to the theory of acoustics, the vibration energy of the radiation plate in the elastic medium excites acoustic waves in the surrounding medium. The vibration characteristics of the radiation plate determine the characteristics of the radiation field. Increase the radiation area or vibration amplitude of the radiation plate can effectively increase the radiation sound power, but in the case where the installation space limits the source size, increasing the vibration amplitude of the radiation plate becomes the focus of the study [2-5]. The material, size, and shape of the radiating panel are factors that affect the vibration amplitude of the radiating panel. In this paper, the five kinds of radiant panels are designed in the same shape and size. Only the effect of material on the vibration amplitude of the radiant panel is taken into account. The finite element simulation and simulation are carried out to provide a strong basis for the design of radiant panels.
\end{abstract}

\subsection{Magnetostrictive transducer structure}

Typical magnetostrictive transducer structure shown in Figure 1., its working principle is that when the excitation coil is switched to an alternating current, a magnetic field is generated in the coil, as a result, Terfenol-D rods generate a magnetostrictive axial motion, which promotes the continuous ultrasonic vibration of the radiant panel, realize the conversion of energy from electrical energy to sound energy [6].

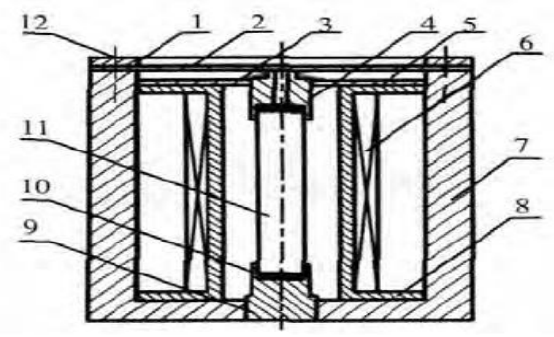

1.Pressure ring 2. Vibration plate 3.Disc spring 4.Guide block 5.Magnet 6.Excitation coil 7.Shell 8.Coil skeleton 9.Adjustment screw 10.Permanent magnet 11.Terfenol-D 12.Screws, washer

Figure 1: Magnetostrictive transducer structure

\section{RADIATION BOARD VIBRATION RESPONSE ANALYSIS}

\subsection{Circular plate theory of vibration}

For the circular plate vibration model shown in Figure 2, existing analytical solutions in elastic mechanics and structural dynamics, let $w$ be the lateral displacement response of the circular plate, then $w$ satisfies $[7,8]$ :

$$
\nabla^{4} \omega+\frac{\rho h}{\partial y^{4}} \bullet \frac{\rho h}{D_{0}} \bullet \frac{\partial^{2} \omega}{\partial t^{4}}=f(x, y, z)
$$

Where $\nabla^{4}$ is a dual-harmonic operator, $\rho$-density, h-Thickness, $D_{0}=\frac{E h^{3}}{12\left(1-\mu^{2}\right)}$-Plate bending stiffness, ${ }^{\mu}$-Poisson's ratio, $w$-Point in the direction of the z-axis displacement, $f(x, y, z)$-Excitation force distributed on a unit area of a radiation plate. 
Derived to solve the equation of amplitude :

$$
w=\left[A J_{0}(k a)+B I_{0}(k a)\right] \cos (n \theta)
$$

k-Wave number $\left.\left(k^{4}=\frac{\rho h \omega^{2}}{D_{0}}\right), \omega_{\text {-Angular frequency }(\omega=2 \pi f}\right)$, $J_{0}$-Zero-order Bessel function, ${ }{ }_{0}$-Zero order imaginary Bessel function

When the radiation plate appears piston vibration type, there is no pitch, pitch line, $n=0$, get the amplitude equation:

$$
w=A J_{0}(k a)+B I_{0}(k a)
$$

Where A and B are arbitrary constants, determined by boundary conditions and initial conditions.

Circular plate vibration sound pressure calculation formula [9]:

$$
P=-\rho c w \omega \sin \omega\left(t-\frac{x}{c}\right)=\rho c u
$$

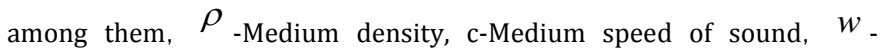
Medium displacement amplitude, $\omega$-Angular frequency, u-Medium particle vibration speed.

Sound field and sound pressure distribution are closely related to displacement $w$, under the same boundary conditions (A and B are equal), same radius a and height $\mathrm{h}$, the displacement $w$ is mainly related to the material selected by the radiant plate $[10,11]$. So, selecting and learning radiation plate materials have great significance for improving the displacement amplitude of the laser plate and increasing the sound pressure amplitude in the resonant cavity.

\subsection{Amplitude and frequency characteristics of radiant panels of different materials}

Table 1: Five kinds of basic mechanical properties of metal materialsmetal materials

\begin{tabular}{|lcll|}
\hline material & densitykg/m3 & $\begin{array}{l}\text { Poisson's } \\
\text { ratio }\end{array}$ & $\begin{array}{l}\text { Young's } \\
\text { Modulus } \\
\text { (Mpa) }\end{array}$ \\
steel & 7800 & 0.285 & 210000 \\
aluminum & 2780 & 0.344 & 71400 \\
copper & 8360 & 0.33 & 117000 \\
brass & 8270 & 0.33 & 92000 \\
greycast iron & 7340 & 0.25 & 120000 \\
\hline
\end{tabular}

Take 5 kinds of common metal material steel, aluminum, copper, brass and HT200 grey cast iron, the basic mechanical properties are shown in Table 1.Through modal analysis, the radiation plate is calculated under the same boundary conditions. The first six modes are shown in the figure. The firstorder modal piston oscillation pattern is an ideal vibration pattern

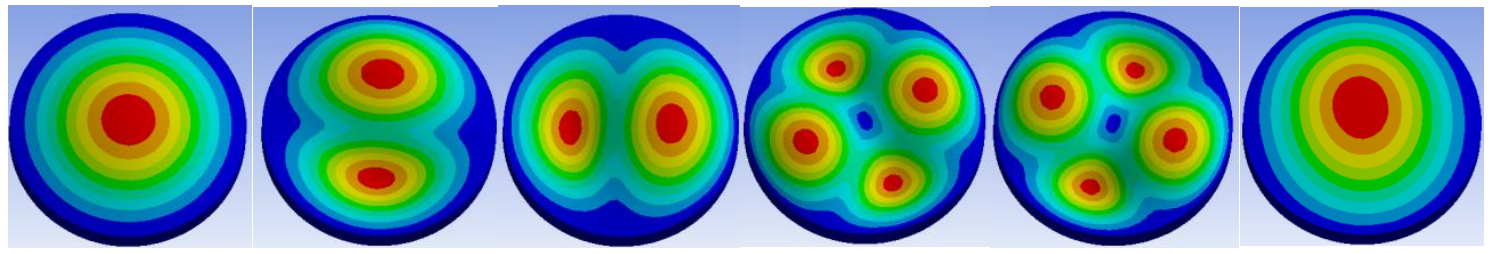

(a) First-order mode (b) Second-order mode (c) Third-order mode (d) Fourth-order mode (e) Fifth-order mode (f) Sixth-order modes

Figure 2: Radiant panel first six order vibration mode

\subsection{Harmonic response analysis}

Harmonic response analysis of 5 materials corresponding radiant panel models using ANSYS Workbench finite element software, simulation of the working process of a radiation plate subjected to continuous sinusoidal excitation of a Terfenol-D rod using a numerical method. A $5 \mathrm{MPa}$ continuous harmonic excitation is applied to a surface with a radius of 10 $\mathrm{mm}$ on the center of the radiation plate. The frequency selection range is $0-20000 \mathrm{~Hz}$ and the step length is $100 \mathrm{~Hz}$. After analysis, the amplitudefrequency distribution curve at the center point of the radiation plate model of different materials is shown in the figure.
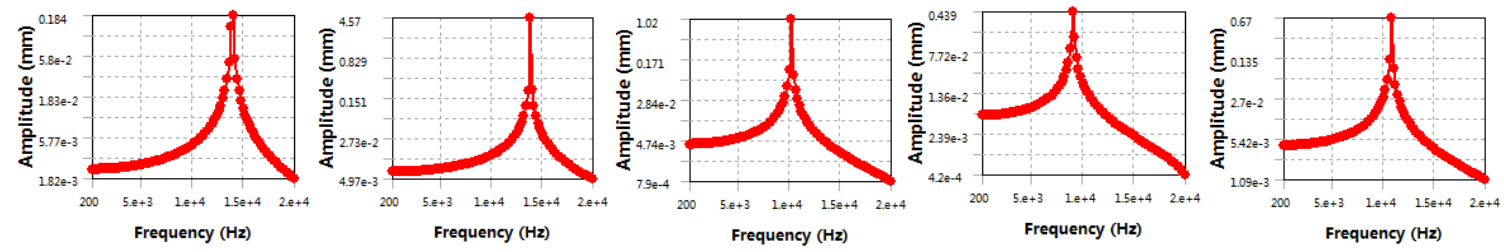

(a) Steel radiation board (b) Aluminum radiation board (c) Copper radiator (e) Brass radiation board (d) Gray cast iron radiation board

Figure 3: Different material corresponding amplitude frequency characteristics of radiant panel

After observing the animation, all four kinds of radiant plates are in the first-order mode and all are piston vibration modes. Extract the corresponding data as shown in Table 2 .
Table 2: Different materials radiant panel model amplitude frequency distribution

\begin{tabular}{|llll|}
\hline Material & frequency/Hz & Vibration ode & Deformation $/ \mathrm{mm}$ \\
\hline steel & 13917 & Axial & 0.184 \\
aluminum & 13809 & Axial & 4.57 \\
copper & 10818 & Axial & 1.02 \\
brass & 9050.2 & Axial & 0.439 \\
grey cast iron & 10769 & Axial & 0.67 \\
\hline
\end{tabular}


Can be seen from Table 2: the radiating plates corresponding to the five kinds of materials have the maximum displacement response at the first

mode frequency of the piston vibration mode, when the five kinds of materials correspond to the radiating plate, the frequency value of the piston vibration mode is reduced by the radiation plate materials steel, aluminum, copper, gray cast iron, and brass. Among them, the frequency values of the radiant plates corresponding to the steel and aluminum materials are all approximately $13900 \mathrm{~Hz}$, brass Corresponding Radiator Frequency Value $9050.2 \mathrm{~Hz}$ Minimum. The output displacement amplitudes of the four types of radiation plates are reduced by the material aluminum, copper, gray cast iron, brass and steel. The output displacement of the aluminum material to the radiation plate is $4.57 \mathrm{~mm}$, it is 4.48-24.8 times the displacement of other kinds of materials. It can be seen that the aluminum radiation plate can output a larger displacement amplitude under high-frequency vibration and is an ideal radiation plate material.

\section{DISTRIBUTION OF SOUND FIELD GENERATED BY RADIATIVE PLATES OF DIFFERENT MATERIALS IN COLUMN-SHAPED ENCLOSED CAVITY}

For the study of the sound field distribution in a closed resonant cavity with a radiant plate at the front end, it is difficult to obtain an analytical solution due to a large number of influencing factors. Using finite element numerical simulation method can effectively solve this problem. Create four kinds of radiation board two-dimensional model in GID software as shown in Figure4, a coustic energy output ends with the same size cylindrical resonant cavity structure, assigning Resonators to Materials in Elastic Materials - STEEL1, radiation board- STEEL1, Gas medium-AIR FLUID; conditions force $\mathrm{F}=1570 \mathrm{~N}$ continuous force is applied to the surface of the radiant panel with a center radius of $10 \mathrm{~mm}$ to impose a fixed constraint on the bottom edge of the resonator, applying a fixed constraint on the bottom edge of the resonator. Problem Data Selected 2D HARMONIC Analysis, select frequency range $1000 \mathrm{~Hz}-20000 \mathrm{~Hz}$ in Interval Date, take $200 \mathrm{~Hz}$ as the step. After Meshing automatically generates the mesh, the model is imported into ATILA Acoustics analysis software for calculation.

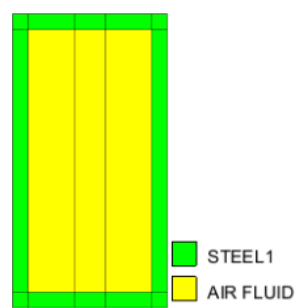

(a)Close

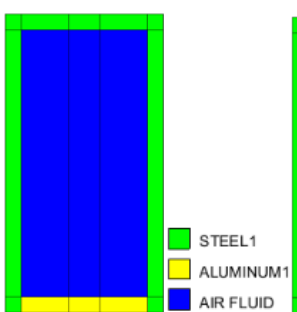

FLUID

(c) Closed cavity3
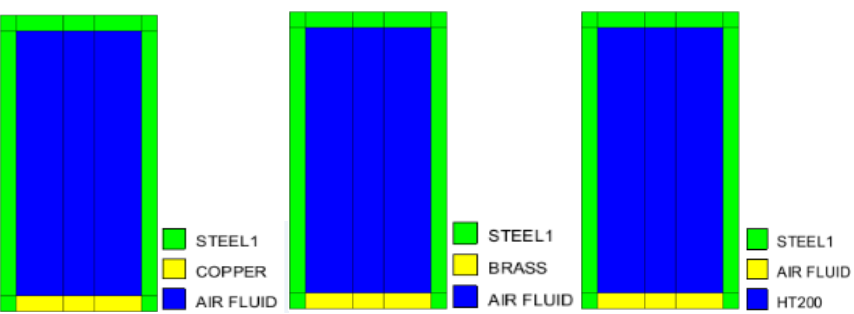

(d) Closed cavity4 (e) Closed cavity5

Figure 4: Closed cavity two-dimensional material model

Figure 5 (a) - (d) shows the distribution of the maximum sound pressure generated in the closed cavity when the resonators are connected to the same size and the closed cavity of the material, and the resonators vibrate at the fundamental frequency respectively. According to the data obtained, it can be seen that when the pulsating pattern of the cavity of the five groups of cavities appears, the frequency is greatly reduced when the standing wave pattern is generated in the cavity and the radiation plates of different materials have a significant effect on the distribution of sound field and pressure changes in the resonant cavity. Observing the animation, it can be seen that the acoustic wave frequencies and amplitudes in the same cross-section of the resonant cavity corresponding to different shapes of radiation plates are all equal, the red and blue bands representing the wave crests and antinodes appear alternately with a phase difference of $180^{\circ}$. This shows that near the fundamental frequency of the resonant cavity, a quasi-wave sound field can be generated in the corresponding resonant cavity of different material radiation plates and the sound pressure amplitude varies greatly. The sound pressure amplitudes produced by the five chamber chambers are reduced by cavity 2 , chamber 4 , chamber 3 , chamber 5 , and chamber 1 . The maximum amplitude of the sound pressure radiated from the aluminum radiant panel is $640.3 \mathrm{~Pa}$, which is $1.2-2.4$ times that of other materials corresponding to the radiated sound pressure of the radiant panel.
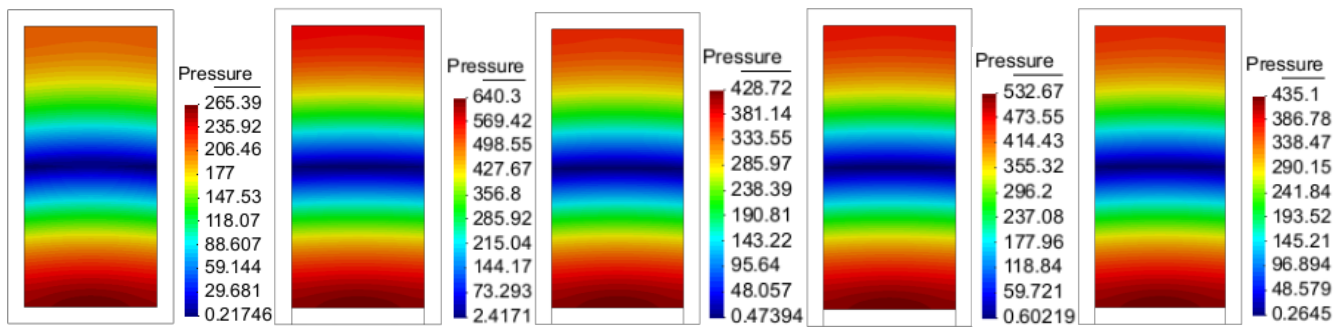

(a) Cavity 1 (b) Cavity 2 (c)Cavity3

(d) Cavity4 (e) Cavity5

Figure 5: Different radiant panel combination structure acoustic press

\section{CONCLUSIONS}

The following conclusions were obtained by numerical simulation of the radiant panel of different shapes.

Under the same excitation condition, when the radiant plates of different materials appeared the piston vibration modes, that the frequency values were successively reduced by steel, aluminum, copper, gray cast iron and brass. Radiant plates of different materials were excited at their respective fundamental frequencies to produce maximum displacement output, the amplitude of the output displacement was successively reduced by aluminum, copper, gray cast iron, brass and steel. The displacement value of the aluminum radiant panel was $4.57 \mathrm{~mm}$, which was $4.48-2.48$ times the output displacement of other materials. It could be seen that the aluminum radiant panel could output large displacement amplitude at high frequency vibration, which was the ideal material for radiant plate.

Under the same excitation force and boundary constraint conditions, the same resonant tube was added, it's the material, size and boundary conditions all affected the sound field distribution, so the vibration frequency of the combination of the resonant cavity and radiation plate was reduced. The standing waves in the five groups chambers were generated at the fundamental frequency, and the amplitude of acoustic

pressure in the cavity is reduced by cavity2, cavity4, cavity3, cavity5, and 
cavity 1 in sequence. The maximum amplitude of the sound pressure radiated from the aluminum radiant panel was $640.3 \mathrm{~Pa}$, which was $1.2-2.4$ times that of the other material radiant panel.

Under the same size, shape, excitation force and boundary conditions, the above analysis results showed that the amplitude of vibration of the aluminum radiant plate and the sound pressure in the same resonant all were the largest. The paper provided favorable basis for the design of radiant plates with high frequency, large amplitude and high sound intensity.

\section{REFERENCES}

[1] Hua, T. 2013. New Type of Ultrasonic Transducer and RadiatorResearch [D]. Shanxi Normal University.

[2] Ming-fan, L. 2006. Low frequency high power application of downhole acoustic generator based on GMM basic research [D]. Zhejiang university.

[3] Ying-tai, Z., Shu-shi, J. 2002. Underwater acoustic transducer and its research and development [J]. Instrument technique and sensor, 11, 46 48.

[4] Klein, E. 1968. Underwater Sound and Naval Acoustical Research and Application befour 1939.The Journal of the Acoustical Society of America, 43 (5), 931-947.

[5] Hueter, T.F. 1987. Twenty Years in Underwater Acoustics:Generation and Reception. The Journal of the Acoustical Society of America, 51 (3B), $1025-1040$
[6] Jian-xin, W., Yao-ning, L., Ling-cong, Z., Xiao-liang, Y. 2015. Magnetostrictive Transducer Radiant Ppanel Displacement Amplification Mmechanism Design [J]. Journal of Mechanical Design and Manufacturing, 03, 201-203+207.

[7] Jian-xin, W., Chang-song, W., Jian-ping, S. 2009. Cylindrical Forced Vibration Regularity of Magnetostrictive Transducer Radiant Panel [J]. Journal of Vibration and Shock.

[8] Zheng-ping, Z., Shu-hong, X., Ji-bao, Q. 2009. Computational Structural Dynamics $[\mathrm{M}]$. Hefei : University of Science and Technology of China Press, 6.

[9] MIP. 2005. The National Defense Science and Technology Rndustrial Nondestructive Testing Personnel Qualification and Certification Training Materials "Eeditorial Committee. Ultrasonic Testing [M]. Beijing : Mechanical Industry Press, 7

[10] Hanafiah, M.M., Ali, M.Y.M., Aziz, N.I.H.A., John, A. 2017. Biogas Production from Agrowaste And Effluents. Acta Chemica Malaysia, 1 (1), 13-15.

[11] Elsayed, J. 2017. Bio-Chemical Biomarkers In Algae Scenedesmus Obliquus Exposed To Heavy Metals Cd, Cu And Zn. Acta Chemica Malaysia, $1(1), 16-20$ 